NASA

Technical Memorandum 107320
Army Research Laboratory Technical Report ARL-TR-1113

\title{
Microelectromechanical Systems for Aerodynamics Applications
}

Mehran Mehregany

Case Western Reserve University

Cleveland, Ohio

Russell G. DeAnna

Vehicle Propulsion Directorate

U.S. Army Research Laboratory

Lewis Research Center

Cleveland, Ohio

Eli Reshotko

Case Western Reserve University

Cleveland, Ohio

Prepared for the

34th Aerospace Sciences Meeting \& Exhibit

sponsored by the American Institute of Aeronautics and Astronautics

Reno, Nevada, January 15-18, 1996

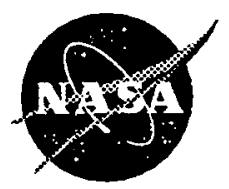

National Aeronautics and

Space Administration

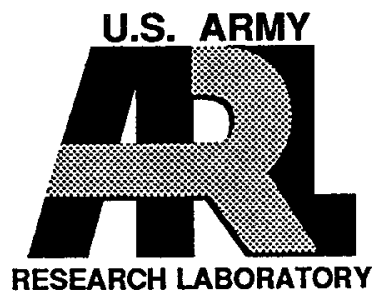




\title{
MICROELECTROMECHANICAL SYSTEMS FOR
}

\section{AERODYNAMICS APPLICATIONS}

\author{
Mehran Mehregany \\ Department of Electrical Engineering and Applied Physics \\ Case Western Reserve University \\ Cleveland, Ohio 44106 \\ Russell G. DeAnna \\ Vehicle Propulsion Directorate \\ U.S. Army Research Laboratory \\ Lewis Research Center \\ Cleveland, Ohio 44135 \\ Eli Reshotko \\ Department of Mechanical and Aerospace Engineering \\ Case Western Reserve University \\ Cleveland, Ohio 44106
}




\section{Abstract}

Microelectromechanical systems (MEMS) embodies the integration of sensors, actuators, and electronics on a single substrate using integrated circuit fabrication techniques and compatible micromachining processes. Silicon and its derivatives form the material base for the MEMS technology. MEMS devices, including microsensors and microactuators, are attractive because they can be made small (characteristic dimension about microns), be produced in large numbers with uniform performance, include electronics for high performance and sophisticated functionality, and be inexpensive. MEMS pressure sensors, wall-shear-stress sensors, micromachined hot-wires are nearing application in aeronautics. MEMS actuators face a tougher challenge since they have to be scaled (up) to the physical phenomena that are being controlled. MEMS actuators are proposed, for example, for controlling the small structures in a turbulent boundary layer, for aircraft control, for cooling, and for mixing enhancement. Data acquisition or control logistics require integration of electronics along with the transducer elements with appropriate consideration of analog-to-digital conversion, multiplexing, and telemetry. Altogether, MEMS technology offers exciting opportunities for aerodynamics applications both in wind tunnels and in flight.

\section{Introduction}

As silicon-chip designers have been packing more electrical elements into smaller packages to make faster computers, others have been building mechanical, chemical, and optical devices on this same silicon. When machines and electronics are combined on a single chip and batch fabricated, the result is a microelectromechanical system (MEMS). ${ }^{1}$ MEMS goes beyond a specific application or device, or a single fabrication process; it provides new and unique capabilities in sensing and actuation. MEMS is a key enabling technology for the development of smart products/systems for a broad range of applications. One advantage of using MEMS over other sensor and actuator technologies is that MEMS can be batch fabricated in large numbers inexpensively (i.e., thousands or millions per batch), making sophisticated distributed sensing and control schemes incorporating large numbers of devices possible. Another advantage is that the small size of MEMS devices (typically less than $1 \mathrm{~mm}$ to as small as a few microns) allows them to be incorporated into a structure without compromising the geometry, weight, function, or inherent properties of the structure. Because of their small size/mass, MEMS devices have higher resonant frequencies than their conventional counterparts.

For fluid dynamics measurements, it is preferred that sensors be small so as to approximate measurement at a point. This is illustrated by recent developments in micro wall-shear-stress sensors and hot-wire anemometers. ${ }^{2}$ Micro-actuators on the other hand have to have a size of the order of a boundary-layer displacement thickness to be properly effective, which in turn allows sub-milli'neter devices near leading edges or at stagnation points, but elsewhere the devices would have to be larger. A microdevice may also be effective if it couples into an unstable or bi-stable phenomenon such as in a fluidic control.

Even when MEMS devices show benefits in the laboratory, there are many packaging challenges for any aeronautics application. Incorporating MEMS into these applications requires new approaches to packaging that goes beyond traditional integrated circuit (IC) packaging technologies. MEMS must interact mechanically, as well as electrically, with their environment, making most traditional chip packaging and mounting techniques inadequate. Aircraft are exposed to natural elements (e.g., rain, sun, ice, insects, and dirt) and human interference (e.g., deicing and cleaning fluids, and maintenance crews). Internal surfaces likely to benefit from MEMS are usually part of the 
propulsion system. Hence, they must operate in environments containing gases with very high temperatures, abrasive particles, and combustion products. Many aerodynamics applications involve curved surfaces and remote access. MEMS devices are often fabricated on stiff and flat silicon substrates and do not easily lend themselves to being mounted on curved surfaces. Thus, flexible substrates and telemetry, along with multiplexing circuits and analog-to-digital conversion is foreseen.

This paper presents an overview of MEMS fabrication and materials. Two sensor examples from the work of the authors are described for a perspective on MEMS capabilities. Issues in fluid dynamics and heat transfer are discussed. A brief discussion of boundary-layer control applications of MEMS sensors and actuators is presented.

\section{Fabrication Technology}

MEMS devices are fabricated by a combination of IC fabrication processes and micromachining techniques. Silicon micromachining represents the fashioning of microscopic mechanical parts from or on a silicon substrate, and is an extension of the IC fabrication technology. Micromachining has been used to, for example, fabricate beams, diaphragms, grooves, orifices, sealed cavities, pyramids, needles, springs, gears, linkages, and motors. With integrated electronics, these microstructures acting as transducer elements have been used to realize a gamut of microsensors and microactuators. ${ }^{3}$

Some of the key silicon micromachining techniques are surface and bulk micromachining, substrate bonding, and micromolding. ${ }^{1}$ In surface micromachining, micromechanical devices are fabricated on the surface of the substrate by consecutive deposition and patterning of thin films of structural and sacrificial materials, with the wafer acting as a mechanical carrier. The structural parts of the device are encased by the sacrificial layers during fabrication. At the end, the sacrificial layers are dissolved in a chemical etchant that does not remove the structural parts. Polysilicon and silicon dioxide are common examples of structural and sacrificial materials, respectively, used in a process known as polysilicon surface micromachining, which is the process used to fabricate the shear stress sensor described later in this paper. Surface micromachining can be used to fabricate conventional beams, diaphragms, and suspensions, as well as more complex mechanical structures such as gears, turbines, and linkages. ${ }^{1}$

Bulk micromachining uses wet and dry silicon etching techniques, with etch masks and etch stops, to sculpt mechanical devices from the silicon wafer. ${ }^{1}$ Certain chemicals, such as $\mathrm{KOH}$, etch the (100) and (110) silicon crystal planes much faster than the (111) planes. This property can be used to create desired shapes/structures by utilizing etch masks and etch stop to selectively expose areas of the silicon wafer to the anisotropic wet etchant. Silicon dioxide and silicon nitride generally have very slow etch rates in the silicon anisotropic wet etchants and make good etch masks. Etch stop regions are created by manipulating the doping concentration to make desired regions resistive to the wet chemical etchant. Silicon pressure sensors, for example, commonly rely at least in part on bulk micromachining technology to create the pressure sensitive diaphragm. Since the geometries that may be fabricated by bulk micromachining are generally limited by the silicon crystal plane orientations (e.g., generally Manhattan planar shapes and sloped side walls) when using wet anisotropic etchants, dry plasma etching of silicon using a reactive ion etcher has been used to define more complex geometries with curved planar features and deep vertical side walls into a silicon wafer. ${ }^{4}$

Bonding techniques permit a silicon substrate to be attached to another substrate, usually glass or silicon, to provide added design flexibility, mechanical support, electrical 
connection, and/or thermal sink/ isolation. ${ }^{1}$ Since the silicon fabrication techniques are generally planar in nature, bonding extends design flexibility in the thickness direction. Electrostatic bonding of silicon to glass substrates and silicon-fusion bonding - bonding of two wafers at high temperature (near $1000^{\circ} \mathrm{C}$ ) in an oxygen or nitrogen ambient - in tandem with bulk machining has been used to fabricate a variety of other MEMS devices (e.g., accelerometers, flow sensor, microvalves, micropumps, etc.). ${ }^{3}$

The aforementioned micromachining techniques are augmented by the capabilities of micromolding, a process in a which a photolithographically-defined mold is used to plate up metallic microstructures. ${ }^{5}$ The mold is dissolved away subsequent to plating to release the metal microstructures. Techniques, such as $\mathrm{x}$-ray photolithography, allow mold features with height-to-width aspect ratios over 100; features a few microns wide and several hundred microns deep with vertical side walls are possible. ${ }^{6}$

\section{Materials Base}

Single-crystalline silicon is generally the basic material for MEMS devices. While semiconductor silicon is well-known for its electronic properties, it is also an excellent mechanical material (e.g., modulus $\sim 190 \mathrm{GPa}$ ) for MEMS applications. However, silicon electronics are limited to operations below $\sim 200^{\circ} \mathrm{C}$, and silicon's excellent mechanical properties start to diminish over $\sim 400^{\circ} \mathrm{C}$. In recent years, silicon carbide (SiC) has emerged as the leading material candidate for applications in high-temperature environments and can complement silicon for high-temperature MEMS applications. With $\mathrm{SiC}$, diodes and more complex electronics have been shown to operate up to $\sim 600^{\circ} \mathrm{C}$, while the excellent mechanical properties of SiC are maintained to much higher temperatures. ${ }^{7}$

In addition to single-crystal silicon, polysilicon, silicon dioxide, and silicon nitride are widely used in MEMS. Other materials which have been or are being experimented with include shape-memory alloy (SMA) metals, tungsten, diamond-like films, piezoelectrics, piezoceramics, polyimides, magnetic or ferrous materials such as nickel, and hightemperature superconductors. While all materials that can be processes with thin film technology can be incorporated into MEMS devices, limitations arise from IC and process compatibility. The former becomes important when an IC facility is used for MEMS fabrication. The latter arises from the fact that a given device fabrication process is a composite of many fabrication steps, all of which must be compatible with respect to materials and processing requirements.

Mechanical properties, such as residual stresses, elastic modulus, fracture stress, adhesion, fatigue, creep, friction, and wear, of thin film materials are important in MEMS development. These properties have been and are being studied to varying degrees. MEMS devices typically have larger surface-to-volume ratio, higher smoothness of the contact surfaces, and smaller contact forces. As a result, conventional wisdom does not always explain the observed behaviors.

\section{Device Examples}

MEMS devices, including both sensors and actuators, are increasingly being studied for aerodynamic applications. The sensor developments are more mature since actuator applications are generally more challenging as pointed out in Introduction. Two sensor examples from the work of the authors are presented in this section to demonstrate the technology. 


\section{Microfabricated Shear-Stress_Sensor}

Many boundary-layer control schemes (discussed later) require knowledge of the surface shear stress. However, the direct measurement of surface shear stress has been elusive. Conventional devices were simply too large or not sensitive enough for practical measurements. A simple and inexpensive direct measurement of surface shear stress can be made using MEMS technology. Microfabricated shear stress sensors based on a floating element balance design, as first demonstrated by Schmidt, ${ }^{8}$ are under development in our laboratory. ${ }^{9}$ Both active and passive designs have been demonstrated, and integration of the sensors with on-chip electronics is pursued. Furthermore, in a collaboration between CWRU and Analog devices, Inc., Waltham, MA, integrated shear stress sensors have been produced which combine surface micromachining with a $2 \mu \mathrm{m}$ CMOS process. These sensors have the transducer element and electronics for signal amplification and conditioning integrated on the same chip.

An SEM photograph of a CWRU active shear stress sensor is shown in Fig. 1. The sensor is fabricated using polysilicon surface micromachining techniques and consists of a rectangular sense plate, $100 \mu \mathrm{m} \times 100 \mu \mathrm{m}$, suspended by $100 \mu \mathrm{m}$-long folded beams. The beams are flexible in the plane parallel to the surface and relatively inflexible in the direction normal to the substrate. The plate and beams are fabricated from $2.2 \mu \mathrm{m}$-thick phosphorous-doped polysilicon. Comb finger structures located on opposite sides of the plate are electrostatic actuators capable of moving the rectangular plate by electrostatic force when a voltage is applied across the outer comb finger pairs.

As gas flows over the chip, the surface shear stress produces a force on the floating element that causes the element to deflect. The deflection of the element is related to the shear force $\mathrm{F}$ and spring constant $\mathrm{k}$ by $\delta=F / k$. As the plate moves, the overlap area between the rectangular plate and a pair of underneath sense electrodes changes, producing a change in capacitance which can be measured with on-chip electronics. Differential pair and charge amplifiers are currently fabricated on-chip in a NMOS process which is combined with the polysilicon surface micromachining process needed to fabricate the transducer element. The electrostatic comb actuators may be used in a feedback control loop to maintain the plate at null position for improved dynamic range and linearity. The actuators also provide the capability for self-calibration and testing. Since the cavity below the plate is exposed to the environment, steady ambient pressure changes have no effect.

To calibrate the sensors, a laminar flow calibration chamber is designed. ${ }^{9}$ The sensor chip is mounted in a recessed groove and subjected to a defined, characterized laminar flow. The gas enters one end of the flow channel, and travels a length sufficient for full flow development before reaching the sensor chip. Pressure ports along the channel allow the pressure gradient of the flow to be characterized. The deflection of the suspended plate is measured under a microscope and the relationship between flow and deflection is aetermined. It is also possible to monitor the element deflection electronically. The shear stress in the flow channel is calculated based on the measured pressure gradient and independently based on the flow rate. The channel height is determined by the thickness of a thin shim stock bolted in between the upper and lower flow channel plates.

The graph in Fig. 2 shows test results for a sample of shear stress sensors. ${ }^{9}$ Sensors with $100 \mu \mathrm{m}$ and $120 \mu \mathrm{m}$ folded beams were tested, illustrating that the sensitivity of the sensor can be tailored by varying the stiffness of the suspended support structure. The suspended plate with the longer support beams is more compliant, exhibiting a higher sensitivity to shear stress. Separate data points indicate test results of different sensors, showing that a similar response is achieved from one sensor to the next. 


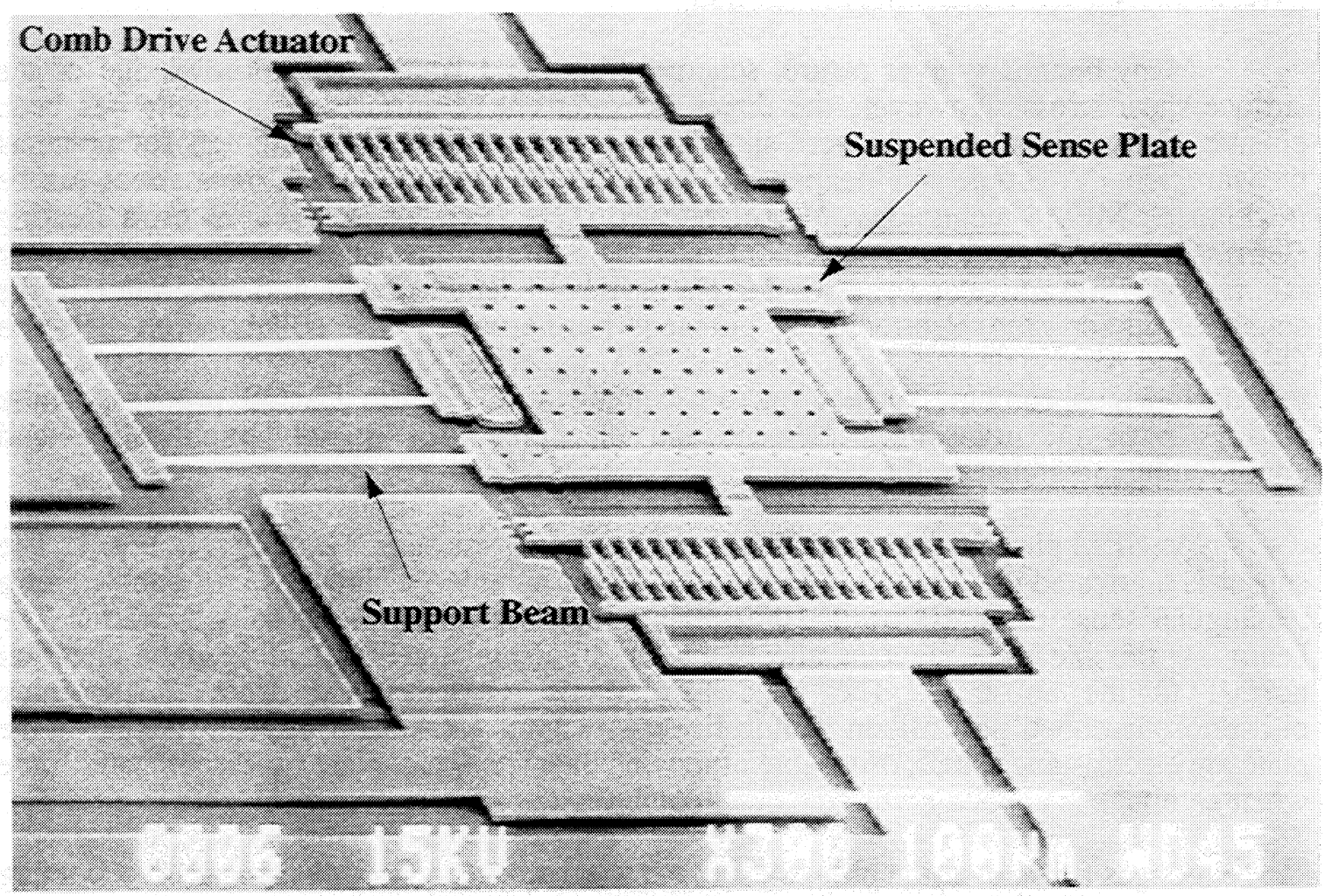

Figure 1: SEM photo of the CWRU shear stress microsensor.

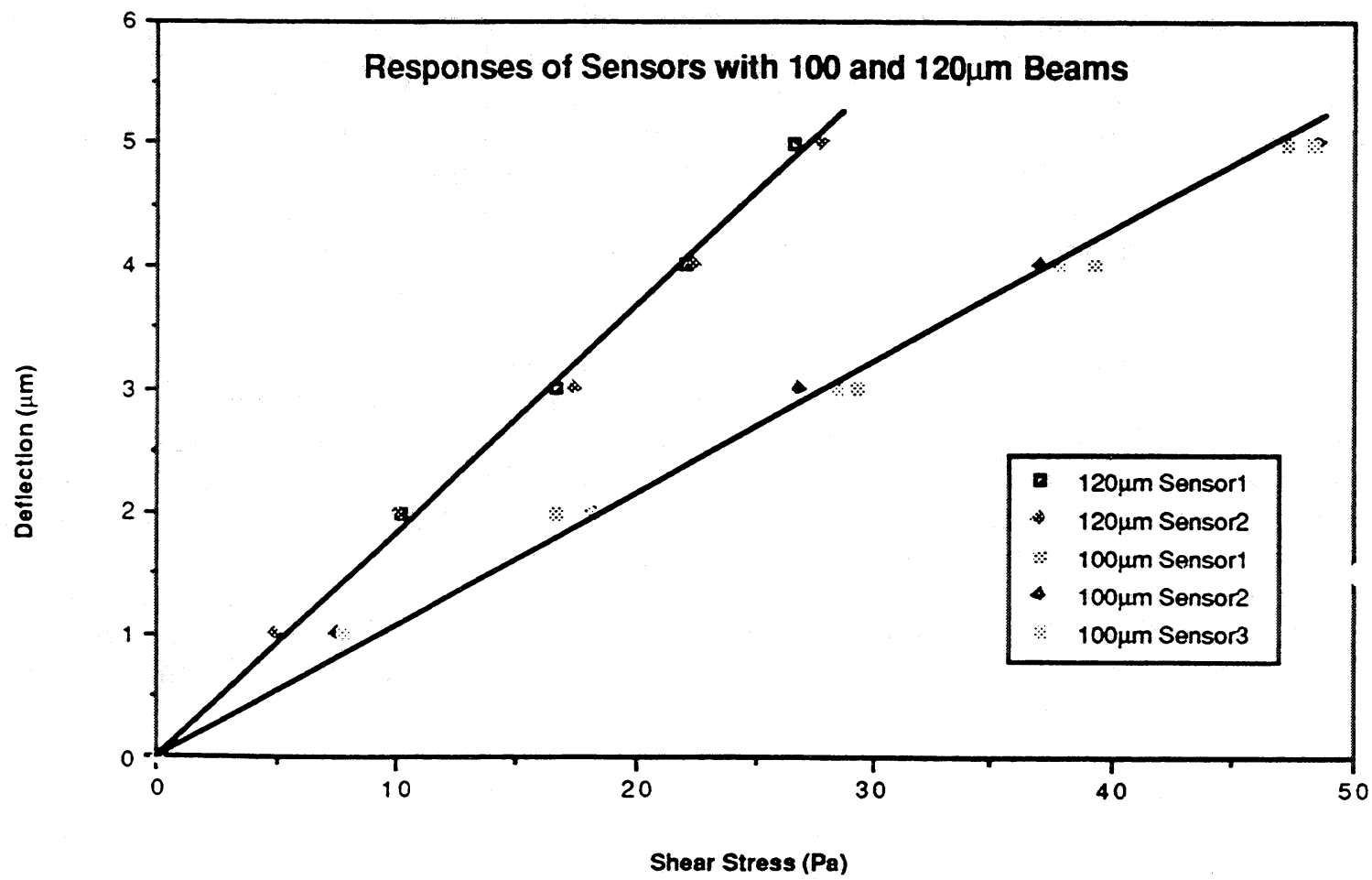

Figure 2: The element deflection as a function of applied shear stress is indicated for three sensors with $100 \mu \mathrm{m}$-long and two sensors with $120 \mu \mathrm{m}$-long support beams. The sensors with longer beams are more compliant, resulting in a greater sensitivity. 
The sensors can also be used to measure the shear stress in a turbulent boundary layer. The devices have resonant frequencies of $\sim 13 \mathrm{KHz}$, but the current electronics have a flat response only to $\sim 3 \mathrm{KHz}$. Clearly, capturing the high-frequency structures in a turbulent flow would require a new mechanical design and new electronics. In turbulent flow, there will be normal pressure fluctuations with magnitudes on the order of $p^{\prime}=0.01\left(1 / 2 \rho V^{2}\right)$. At $\operatorname{Re}_{\theta}=1000$, the pressure fluctuations are 2.2 times larger than the shear stress; at $\operatorname{Re}_{\theta}=50,000$, the ratio is 5.8. In addition to the large squeeze damping effect between the floating plate and the substrate, the stiffness of the beams supporting the plate in the normal direction is a factor of $\sim 10$ times higher than that in the lateral direction, so these normal loads should not ruin the sensor's performance.

\section{Heat Flux Sensor Arrays in a Smart Skin Technology}

In many applications, distributed sensors, actuators, and electronics acting over a large surface (larger than typical IC chip dimensions) with arbitrary shapes are desired. For example, it may be desirable to place an array of sensors, actuators, and electronics on the curved surface of an aircraft wing. MEMS devices are currently fabricated on rigid substrates that are not suitable to mounting on curved surfaces, making many applications impractical. In the case of the aircraft wing, small recesses would need to be machined in the wing along with wire through-holes at each distributed point. To solve this problem, suppose that a thin, flexible, large area sheet could be manufactured containing arrays of MEMS, including electronics. Such a sheet could be manufactured separately in bulk and applied directly to an arbitrary structural surface, providing a convenient means for sensing, signal processing, and actuation for a wide variety of structures. This smart skin technology is under development at Advanced MicroMachines Incorporated (AMMi), Cleveland, $\mathrm{OH}$. A heat flux sensor array for measuring heat transfer phenomenon in turbomachinery is being developed that also serves as a vehicle for developing smart skin technology. The concept is outlined in Fig. 3. An array of heat flux sensors is contained in a flexible surface that is sized to conform over a turbine blade or other test article. Electrical connections are made by metal lines microfabricated in the flexible material, eliminating the need for external wires. At one end of the array, which may be extended before the base of the turbine blade and away from the turbine gases, an interface chip is directly mounted to contact pads in the flexible material, eliminating the need for external wiring. The interface chip provides signal amplification and substantially reduce the number of output lines required through multiplexing. The sensing array is bonded to the surface of the turbine blade, conforming to its shape and providing multiple sensor locations distributed across the surface. The skin sensor array will allow a detailed area profile of surface heat flux to be measured, providing valuable data about unsteady heat transfer phenomenon in turbomachinery.

A cross section of an individual heat flux sensor is shown in Fig. 4. The sensor is comprised of temperature sensitive metal resistors sputtered and patterned on a flexible substrate. The insulating materials are spin-coated and patterned. During processing, the flexible substrate is mounted on a silicon wafer to provide rigidity and semiconductor material handling compatibility. After the sensors are completed, the flexible substrate is removed from the silicon wafer. The sensor employs a bridge arrangement of four temperature sensitive resistors. One resistor pair has a thin coating of insulation, while the other has a thick coating. As heat flows through the sensors into the test article, there is a slight temperature difference between the resistor pairs due to the larger thermal resistance over the resistors with thicker insulation. This temperature difference is proportional to the heat flux. The temperature change in the resistors produces a change in resistance that 
imbalances the bridge. If a current flows through the bridge, there will be a voltage between the output nodes that is proportional to the heat flux.

(a)

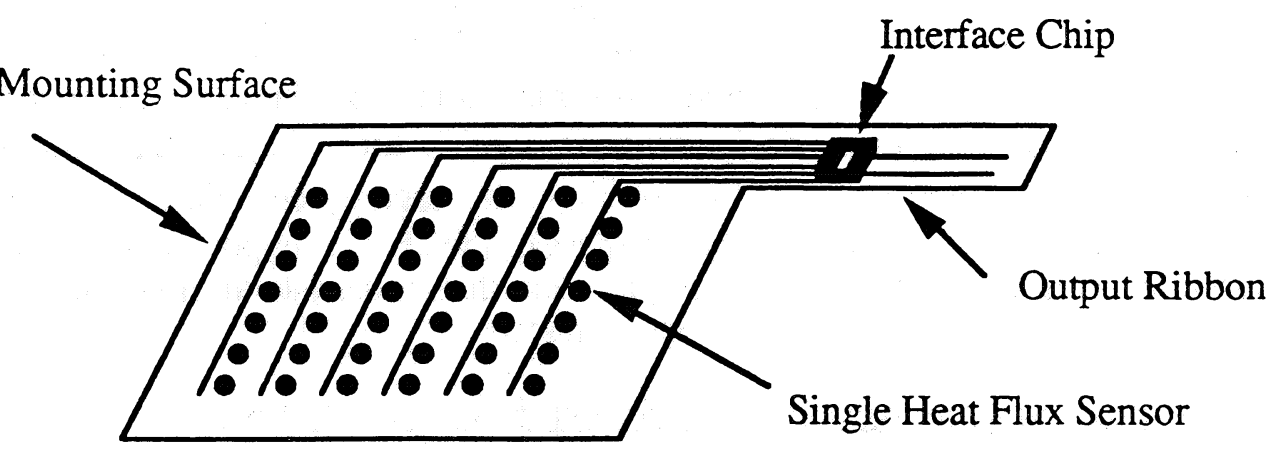

(b)

Figure 3: Heat flux sensor array system on a flexible skin: (a) schematic; and (b) actual.

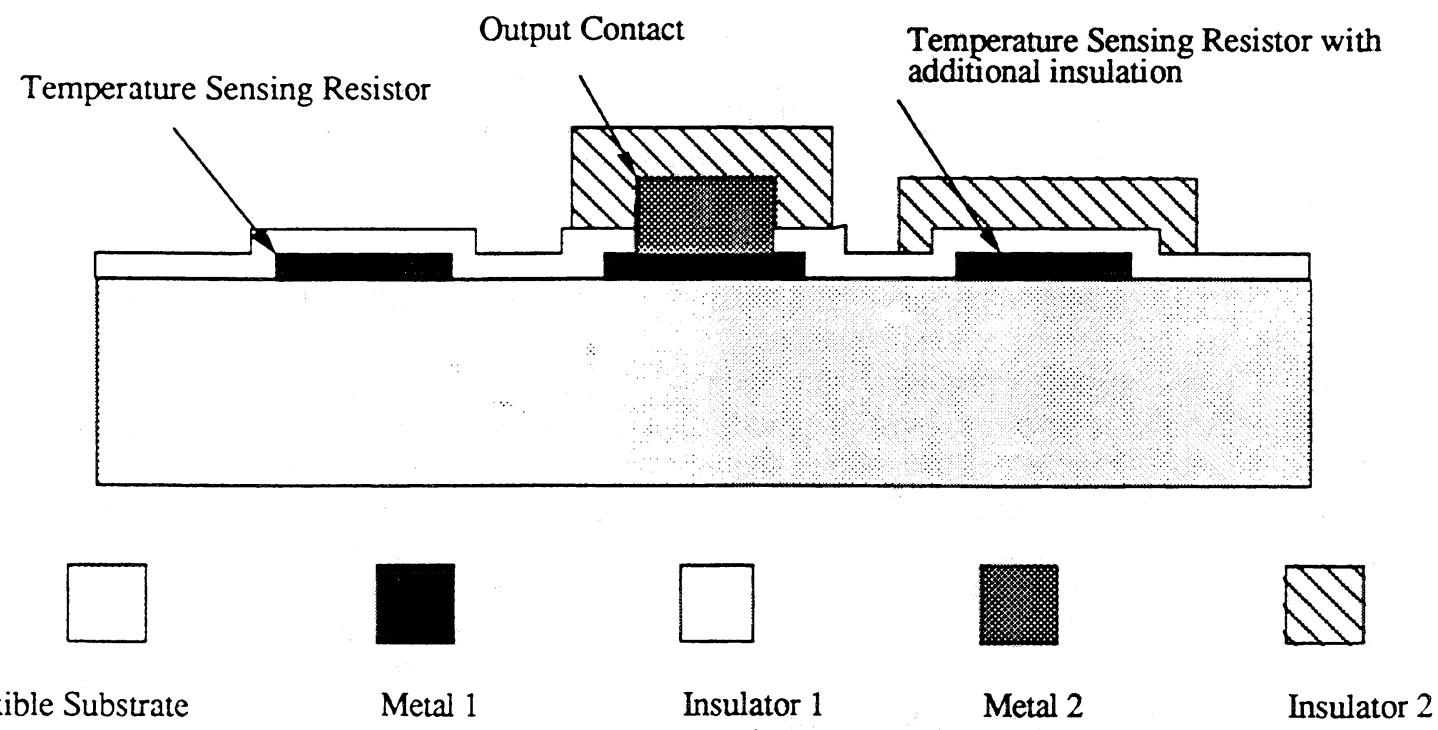
Figure 4: Cross section of a single heat flux sensor. 
In addition to the heat flux sensing application described here, we envision a wide range of MEMS that could be employed in a smart skin for a wide variety of surface sensing and actuation applications. Incorporation of such devices into a smart skin array would further extend the capabilities of sensor technologies for use in smart structures by providing a convenient means of sensor placement on an arbitrary structure surface.

\section{Issues In Fluid Dvnamics And Heat Transfer At Micro Scales}

Because of the low Reynolds (Re) numbers associated with micromachines, viscous forces dominate inertial forces, Stokes flow is likely, the flow is often reversible in direction, and friction is proportional to velocity - instead of velocity squared as in inertial-dominated flows. The entrance region in microchannels is short, while microvalves, pumps, and jets operate with length-to-diameter ratios less than one, where fully-developed profiles are not likely. In all low-Re-number flows, the friction coefficient is inversely proportional to length.

\section{Continuum Assumption}

At standard atmospheric conditions, the continuum assumption may fail when the size of the device approaches the micrometer range. For devices with characteristic length around $1 \mu \mathrm{m}$, the Knudsen number, defined as the ratio of mean-free path to the geometric length scale, would be 0.07 at STP. For Knudsen numbers above 0.01 , the continuum approach may be inadequate since the flow no longer experiences sufficient collisions to behave according to the equations governing macro flows. Instead, rarefied-gas methods must be used.

\section{Turbulent Boundary-Laver Roughness}

In a turbulent boundary layer, the viscous sublayer is defined by $y^{+}=y u_{*} / v<5$. Where $u_{*}=\sqrt{\tau_{w} / \rho}$. For an actuator with height $\mathrm{H}^{+}$less than 5 , the flow is hydraulically smooth, and friction drag is not increased. For $5 \leq H^{+} \leq 70$, the friction coefficient depends on Re number - decreasing with larger Re number. And for $H^{+} \geq 70$, the flow is considered fully rough, and the friction coefficient is independent of $\mathrm{Re}$. With air at $300 \mathrm{~m} / \mathrm{sec}$ (Mach number approaching one) and $1 \mathrm{~m}$ downstream from the leading edge at STP, the flow is dynamically smooth for any device with height less than $13 \mu \mathrm{m}$, transitional between $13 \mu \mathrm{m}$ and $182 \mu \mathrm{m}$, and fully rough above $182 \mu \mathrm{m}$.

\section{Heat Transfer}

In heat transfer, the Biot Number (Bi) represents the ratio of surface convection to conduction within a body. In a body of th: kness $L$ and thermal conductivity $k, B i$ is $B i=h L / k$. The small length scales in MEMS yield small Biot numbers. These devices will be relatively uniform in temperature; lumped-capacity, thermal analysis suffices, and thermal stresses will be small. For example, a $10 \mu \mathrm{m}$-thick silicon membrane in a field with heat-transfer coefficient $h=5000 \mathrm{~W} / \mathrm{m}^{2} /{ }^{\circ} \mathrm{C}$ would give $\mathrm{Bi}=3 \times 10^{-4}$. In unsteady heat transfer, the Fourier number ( $\mathrm{Fo}$ ) represents the ratio of the heat conduction rate to the rate of thermal energy storage in a solid. For a device with characteristic length $L$, thermal diffusivity $\alpha$, and unsteady time scale $\mathrm{T}$, the Fourier number is $F_{o}=\alpha \mathrm{T} / L^{2}$. In MEMS, the Fourier number will be large, thermal energy storage is small, and the device will be able to capture high-frequency phenomena. A $10 \mu \mathrm{m}$-thick silicon membrane, would have $\alpha / L^{2}$ equal to $1 \mathrm{MHz}$. In heat transfer with natural convection, the Grashof number $(\mathrm{Gr})$ 
represents the ratio of buoyancy to viscous forces. For a device at temperature $T_{w}$, $G r=\frac{g \beta\left(T_{w}-T_{0}\right) L^{3}}{v^{2}}$. Gr will normally be very small, and buoyancy is negligible. Natural convection of heat will be small, and a viscous layer dominated by thermal diffusion will occur.

\section{Surface Tension}

Surface tension is important whenever there is an interface between a gas, solid, and liquid. The pressure change across an interface with curvature $R$ is:

$$
\Delta p=2 \gamma \cos \theta / R,
$$

where $\mathrm{R}$ is the channel radius, $\gamma$ is the surface tension, and $\theta$ is the contact angle. Thus, pressure difference increases with shrinking dimensions. For a $20 \mu \mathrm{m}$ diameter channel with a water-air interface, the maximum pressure change across the interface is $1400 \mathrm{~Pa}$. This corresponds to a pumping height of $10 \mathrm{~cm}$ of $\mathrm{H}_{2} \mathrm{O}$. Surface tension in small channels $(1 \mu \mathrm{m})$ can greatly affect flow rate. For example, alcohol and water will have greatly different flow rates in tiny passages when an interface is present because of their surface tension differences.

\section{Boundary-Layer Control}

Boundary-layer control involves introducing an actuator or process which causes the boundary layer to diverge from its natural development. Control may require an outside source of power - active control; or it may be entirely passive. If the control input is based on continuous measurements, the control is considered to be interactive with either an open control or closed-loop feedback control. ${ }^{10}$

Any application of an active system must consider the additional energy used to power the actuators, sensors, and electronics, as well as any added cost and complexity. This is an area where interactive MEMS may be able to reduce drag by local manipulation of the quasiperiodic, near-wall, boundary-layer events. Systems consisting of arrays of sensors and actuators could manipulate certain organized structures (spatial and temporal gradients in vicinity of the sub-millimeter and millisecond scale) occurring randomly within the boundary layer. ${ }^{11}$ MEMS is not likely to benefit laminar boundary layers because traditional control methods using global fluid injection/suction or heating/cooling without local measurements or feedback would be more efficient.

The near-wall events $\left(0 \leq y \leq 100 v / u_{0}\right)$ in a turbulent boundary layer have counterrotating, streamwise vortices having diameters approximately $40 v / u$ and freq'uency scales of the order $0.0035 u_{*}^{2} / v .^{11,12}$ This corresponds to structures of size $100 \mu \mathrm{m}$ occurring at $31 \mathrm{KHz}$ in a $300 \mathrm{~m} / \mathrm{sec}$ flow at $1 \mathrm{~m}$ from the leading edge.

\section{Skin-Friction Reduction}

A turbulent boundary layer is less prone to separation but is characterized by large skin friction. One technique for reducing the turbulent skin friction is to reduce the nearwall momentum by blowing. The local skin-friction coefficient for a flat plate is: 


$$
C_{f}=2 \frac{d \delta_{\theta}}{d x}-2 \frac{V_{0}}{U_{\infty}}
$$

where $V_{0}$ is the normal velocity of fluid injected through the surface (positive in blowing). Because blowing reduces the skin-friction drag but increases the pressure drag by tending to separate the boundary layer, the goal is to reduce friction while avoiding actual separation. Arrays of microjets and shear-stress sensors could be used. The sensors would monitor the local shear stress and control the amount of blowing to avoid separation.

\section{Wave Cancellation to Delay Transition}

If the frequency, orientation, and phase angle of the dominant element of the spectrum of growing linear disturbances (T-S wave) in the boundary layer is detected, a control system and appropriately located disturbance generators may then be used to effect a desired cancellation or suppression of the detected disturbances by introducing a disturbance of opposite phase. ${ }^{15}$ MEMS wall actuators could provide wall heating/cooling, plate vibration, or periodic suction/blowing. This is very similar to active-noise-control systems. In noise cancellation, an array of inexpensive MEMS microphones could be used to measure the spatial and temporal noise. This data could then be combined with neural networks controlling macro-scale actuators to cancel the noise.

\section{Aircraft Control}

Microflaps attached to the leading edge of an F-15 fighter have been proposed for creating rolling moments. ${ }^{14}$ These flaps are located near the leading edges of highly swept delta wings. When activated on one side, they alter the leading edge vortex on that side of the wing creating an imbalance in the vortex lift between the two sides of the wing, thus giving rise to the rolling moment. The resulting rolling moment obtained in the reported experiments ${ }^{9}$ is of the order $1 \%$ of the "vortex lift moment" defined as the products of the vortex lift force on one side of the wing times the distance between the point of application of the moment and the delta-wing centerline. A larger flap closer to the leading edge gave a larger result.

\section{Conclusion}

MEMS signifies the integration of sensors, actuators, and electronics on a single substrate. Because of their small size, low cost, high performance, and sophisticated functionality, MEMS sensors and actuators offer exciting opportunities for aerodynamics applications. MEMS sensor development for aerodynamic applications is more mature than that of the actuator development, since MEMS actuators have to be scaled (up) to the physical phenomena that are being controlled. While laboratory demonstrations of MEMS devices are orten promising, significant packaging challenges must usually be overcome for practical use.

\section{Acknowledgment}

This paper includes results from research contracts sponsored by ARO, AFOSR, and NASA at CWRU and AMMi. 


\section{References}

[1] M. Mehregany, "Microelectromechanical Systems," IEEE Circuits and Devices, pp. 14-22, July 1993.

[2] F. Jiang, Y.-C. Tai, C.-M. Ho, and W. Li, "A Micromachined Polysilicon Hot-Wire Anemometer," in Technical Digest, IEEE Solid State Sensor and Actuator Workshop, Hilton Head, SC, June, 1994, pp. 264-267.

[3] Technical Digests, International Conferences on Solid State Sensors and Actuators, 1983, 1985, 1987, 1989, 1991, 1993, and 1995.

[4] E. Klaassen, K. Petersen, J. Noworolski, J. Logan, N. Maluf, J. Brown, C. Storment, W. McCulley, and G. Kovacs, "Silicon Fusion Bonding and Deep Reactive Ion Etching; A New Technology for Microstructures," in Technical Digest, The 8th Int. Conf. on Solid State Sensors and Actuators, Stockholm, Sweden, June 1995.

[5] H. Miyajima and M. Mehregany, "High-Aspect-Ratio Photolithography for MEMS Applications," J. of Microelectromechanical Systems, vol. 4, pp. 220-229, Dec. 1995.

[6] H. Guckel, T. Christenson, K. Skrobis, J. Klein, and M. Karnowsky, "Design and Testing of Planar Magnetic Micromotors Fabricated by Deep X-ray Lithography and Electroplating," in Technical Digest, The 7th Int. Conf. on Solid State Sensors and Actuators , Yokohama, Japan, June 1993.

[7] G. Krotz, W. Legner, C. Wagner, H. Moller, H. Sonntag, and G. Muller, "Silicon Carbide as a Mechanical Material," in Technical Digest, The 8th Int. Conf. on Solid State Sensors and Actuators, Stockholm, Sweden, June 1995.

[8] M. Schmidt, R. Howe, S. Senturia, and J. Haritonidis, "Design and Calibration of a Micro-fabricated Floating-Element Shear-Stress Sensor," IEEE Trans. Elect. Dev., vol. 35, pp. 750-757, June 1988.

[9] T. Pan, D. Hyman, M. Mehregany, E. Reshotko, and B. Willis, "Characterization of Microfabricated Shear Stress Sensors," in Technical Digest, The 8th Int. Conf. on Solid State Sensors and Actuators, Stockholm, Sweden, June 1995.

[10] S. Wilkinson, "Interactive Wall Turbulence Control," in Viscous Drag Reduction in Boundary Layers, eds., D. Bushnell and J. Hefner, vol. 123, Progress in Astronautics and Aeronautics, AIAA, Washington, DC, 1990, pp. 479-509.

[11] M. Gad-el-Hak, "Interactive Control of Turbulent Boundary Layers: A Futuristic Overview," AIAA Journal, vol. 32, pp. 1753-1765, Sept. 1994.

[12] R. Blackwelder and J. Haritonidis, "Scaling of the Bursting Frequency in Turbulent Boundary Layers," J. of Fluid Mechanics, vol. 132, 1983, pp.87-103.

[13] X.Fan, T. Herbert, and J. Haritonidis, "Transition Control With Neural Networks," AIAA Paper 95-0674, 1995.

[14] C. Liu, T. Tsao, Y.-C. Tai, T.-S. Leu, C.-M. Ho, W.-L. Tang, and D. Miu, "OutOf-Plane Permalloy Magnetic Actuators for Delta-Wing Control," in Proceedings, IEEE Workshop on Microelectromechanical Systems, Amsterdam, The Netherlands, Jan., 1995, pp. 7-12. 
Public reporting burden for this colection of information is estimated to average 1 hour per response, including the time for reviewing instructions, searching existing data sources, Peoction of information. Send comments regarding this burden estimate of any other aspect of this colection of information, including suggestions for reducing this burden, to Washington Headquarters Services, Directorate for Information Operations and Reports, 1215 Jefler Davis Highay, Suite 1204, Arlington, VA 22202-4302, and to the Office of Management and Budget. Papenwork Reduction Project (0704-0188), Washington, DC 20503.

\begin{tabular}{l|l|l}
\hline 1. AGENCY USE ONLY (Leave blank) & 2. AEPORT DATE & 3. REPORT TYPE AND DATES COVERED
\end{tabular}

\begin{tabular}{|l|c|c|}
\hline 1. AGENCY USE ONLY (Leave blank) & $\begin{array}{c}\text { 2. AEPORT DATE } \\
\text { September } 1996\end{array}$ & $\begin{array}{r}\text { 3. REPORT TYPE AND DATES COVERED } \\
\text { Technical Memorandum }\end{array}$
\end{tabular}

4. TITLE AND SUBTITLE

5. FUNDING NUMBERS

Microelectromechanical Systems for Aerodynamics Applications

WU-505-62-ON

6. AUTHOR(S)

1L161102AH45

Mehran Mehregany, Russell G. DeAnna, and Eli Reshotko

8. PERFORMING ORGANIZATION REPORT NUMBER

NASA Lewis Research Center

Cleveland, Ohio 44135-3191

and

E-10417

Vehicle Propulsion Directorate

U.S. Army Research Laboratory

Cleveland, Ohio 44135-3191

9. SPONSORING/MONITORING AGENCY NAME(S) AND ADDRESS(ES)

National Aeronautics and Space Administration

Washington, D.C. $20546-0001$

and

U.S. Army Research Laboratory

Adelphi, Maryland 20783-1145

10. SPONSORINGMONITORING AGENCY REPORT NUMBER

NASA TM-107320

ARL-TR-1113

11. SUPPLEMENTARY NOTES

Prepared for the 34th Aerospace Sciences Meeting \& Exhibit sponsored by the American Institute of Aeronautics and Astronautics, January 15-18, 1996, Reno, Nevada. Mehran Mehregany, Department of Electrical Engineering and Applied Physics, Case Western Reserve University, Cleveland, Ohio 44106; Russell G. DeAnna, Vehicle Propulsion Directorate, U.S. Amy Research Laboratory, Lewis Research Center, Cleveland, Ohio 44135; Eli Reshotko, Department of Mechanical and Aerospace Engineering, Case Western Reserve University, Cleveland, Ohio 44106. This paper includes results from research contracts sponsored by ARO, AFOSR, and NASA at CWRU and AMMi. Responsible person, Russell G. DeAnna, organization code 0300, (216) 433-3385.

12a. DISTRIBUTION/AVAILABILTTY STATEMENT 12b. DISTRIBUTION CODE

Unclassified -Unlimited

Subject Category 06

This publication is available from the NASA Center for AeroSpace Information, (301) 621-0390.

13. ABSTRACT (Maximum 200 words)

Microelectromechanical systems (MEMS) embodies the integration of sensors, actuators, and electronics on a single substrate using integrated circuit fabrication techniques and compatible micromachining processes. Silicon and its derivatives form the material base for the MEMS technology. MEMS devices, including microsensors and microactuators, are attractive because they can be made small (characteristic dimension about microns), be produced in large numbers with uniform performance, include electronics for high performance and sophisticated functionality, and be inexpensive. MEMS pressure sensors, wall-shear-stress sensors, micromachined hot-wires are nearing application in aeronautics. MEMS actuators face a tougher challenge since they have to be scaled (up) to the physical phenomena that are being controlled. MEMS actuators are proposed, for example, for controlling the small structures in a turbulent boundary layer, for aircraft control, for cooling, and for mixing enhancement. Data acquisition or control logistics require integration of electronics along with the transducer elements with appropriate consideration of analog-to-digital conversion, multiplexing, and telemetry. Altogether, MEMS technology offers exciting opportunities for aerodynamics applications both in wind tunnels and in flight.

14. SUBJECT TERMS

MEMS; Sensors; Actuators; Aerodynamics

15. NUMBER OF PAGES

14

6. PRICE CODE

$\mathrm{A03}$

17. SECURTY CLASSIFICATION

18. SECURTYY CLASSIFICATION

19. SECURITY CLASSIFICATION OF ABSTRACT OF REPORT

Unclassified OF THIS PAGE

Unclassified

Unclassified

NSN 7540-01-280-5500

Standard Form 298 (Rev. 2-89)

Prescribed by ANSI Std. Z39-18

20. LIMITATION OF ABSTRACT 